\title{
Five days daily sessions of noninvasive blood glucose level predictions based on amplitude modulated ultrasound and infrared technique over a healthy and diabetic subject.
}

\author{
Md Koushik Chowdhury ${ }^{1 *}$, Anuj Srivastava ${ }^{1}$, Neeraj Sharma ${ }^{1}$, Shiru Sharma ${ }^{1}$ \\ ${ }^{I}$ School of Biomedical Engineering, Indian Institute of Technology (Banaras Hindu University), Varanasi, \\ Uttar Pradesh, India. \\ *Email: kchoudhary.rs.bme11@itbhu.ac.in
}

\begin{abstract}
A noninvasive method based blood glucose predicting device with medically acceptable readings could transform the diabetic management protocols. Noninvasive blood glucose monitoring devices will increase patient compliances along with reduction of medical burden and its related expenses. Here, we had utilized the indigenously developed amplitude modulated ultrasound and infrared technique based noninvasive glucometer for this purpose. A lab based study had been performed to measure the performance of the modulated ultrasound and infrared technique based noninvasive glucometer. A total of 02 subjects ( 01 healthy normal subject and 01 diabetic subject) were engaged in this study. The working accuracy of noninvasive glucometer readings had been compared with the invasive glucometer readings and plotted over the Clarke Error Grid for its critical analysis. The experimental result depicts a good relationship exists between the predicted (noninvasive) and reference (invasive) blood glucose levels. The Clarke Error Grid Analysis depicts that results of the predictions as well as the reference measurement values occupies the medically significant $A$ and B domains. This experimental result directs towards the potential applications of amplitude modulated ultrasound and infrared technique for continuous noninvasive blood glucose level predictions.
\end{abstract}

Keywords: blood glucose, noninvasive, invasive, amplitude modulated ultrasound, infrared technique.

\section{Introduction}

At present 38.2 crores diabetic patients (age 40-59 years) exists worldwide. Moreover, $80 \%$ from them are the residents of middle as well as lower income territories [1-3]. Internationally, different types of diabetes are on expansion mode, with particular major expansion of population belonging to Type II diabetic diseases [13]. Various predictions indicate that the diabetic population will expand by 55\% in near future (2035) [1]. Furthermore, 2.1 crores medical cases of pregnancy in the year 2013, along with elevated blood glucose conditions were diagnosed, only to increase medical burden at the international level [1]. The impact of diabetes is expensive and deadly. Diabetes related health issues had claimed 51 lakh lives worldwide in 2013 with massive 54,800 crores dollar expenditures [1]. As of 2013, diabetic population count for our country, from the age group (20-79) years estimates around 6.51 crores peoples [1]. It will be 10.9 crores diabetic peoples by the year 2035. During the year 2013, 11 lakh people died in India due to diabetes related causes [1-3].

All this data and information directs towards tight and robust management of diabetic conditions [1-5]. The invasive blood glucose detecting glucometer provided diabetic peoples with tight vigilance over blood glucose level management [4-6]. The invasive glucometer comes with test strips, its respective measuring devices and the sharp pointed needle based equipment for skin-tissue puncturing procedures [4-6]. Small drop of human blood are needed to perform the electrochemical reactions to measure the blood glucose levels [4-6]. These medical devices are fast and reliable [4-6]. But some medical and clinical research studies pointed towards certain issues like (i) Practice of invasive mode for frequent tissue puncturing, (ii) Skin infections related issues, (iii) Repeated puncturing of skin to vary the permeability factors which influences the tissue analytes [4-6]. To find the better alternative, researchers had approached for noninvasive technology for blood glucose level predictions [5-7]. Various optical and other technique based noninvasive approaches are as follows: Infrared absorption [7-9], Near Infrared scattering [7,10], Raman spectroscopy [7,11], Fluorescent Technology [7,12], Thermal gradient spectroscopy [7,13], Polarimetry [7, 14], Polarization heterodyning [15], Photonic crystal [16], photo acoustic technology [7, 17], Photo thermal [18], Optical Coherence Tomography (OCT) techniques [7, 19], Ultrasound-modulated optical technique [20] and Occlusion spectroscopy [21]. Motivated by the optical techniques [22] based approaches we had utilized amplitude modulated ultrasound and infrared techniques to detect the blood glucose levels in noninvasive manner during 05 days daily sessions on one healthy normal subject and one diabetic subject respectively. 
Rest of the Research Article had been organized as follows: Section II includes Materials and methodology, Section III consists of Results and discussion portions. Section IV concludes the Research Article, which is followed by the Acknowledgment and the Reference portions.

\section{Noninvasive method based blood glucose level predictions:}

\section{Materials and Methodology}

\section{Indigenously designed and developed noninvasive MUS-IR unit principle of working for blood glucose} level predictions

Under this methodology we had applied amplitude modulated ultrasonic standing waves and infrared LED (Light Emitting Diode) light source-detector assembly for blood glucose level predictions by noninvasive approach. When ultrasonic sound wave passes through the living biological blood tissue complex medium of origin, it introduces vibrational patterns into that respective medium [23-32]. Actually, when two oppositely moving ultrasonic travelling waves were combined, the ultrasonic standing waves were generated. These typical waves were originated from a pair of transducers or one transducer and its reflector [23-32]. Standing waves represents maximum (antinodal) and minimum (nodal) values just double over a length of a single wavelength [23-32]. Within the propagating segment a discontinuities like molecules attains the arrangement-based acoustic potential energy owing to its existence in the particular ultrasonic filed. Moreover the suspended molecules tend to locate and concentrate at the point of lowest acoustic potential energy [23-32]. For the molecules, these regions are generally near to pressure nodes, which are distanced from each other by a space of half a wavelength [23-32]. Similarly, when molecular diameters were smaller then the ultrasonic wavelength, the most important force of radiation $\left(\mathbf{F}_{\mathbf{r}}\right)$ applied over the molecular volume $\left(\mathbf{V}_{\mathbf{c}}\right)$ located by path length of $(\mathbf{z})$ from the pressure node had been represented from the gradient of the molecular acoustic potential energy and provided in Equation No.1 as shown below:

$$
F_{r}=-\left[\frac{\pi p_{0}^{2} v_{c} \beta_{w}}{(2 \lambda)}\right] \cdot \phi(\beta, \rho) \cdot \sin (4 \pi z / \lambda)
$$

Where,

$\mathbf{z}=$ distance under measurement.

$\mathbf{P}_{\mathbf{0}}=$ crest amplitude of the ultrasonic waves under application.

$\lambda=$ wavelength of ultrasonic standing waves.

When the concepts of compressibility factors $\left(\boldsymbol{\beta}_{\mathrm{w}}\right)$ for the suspending segments were considered [23-32], the phenomenon had been expressed as follows:

Where,

$$
\phi(\boldsymbol{\beta}, \boldsymbol{\rho})=\left[\frac{5 \rho_{\mathrm{c}}-2 \rho_{\mathrm{w}}}{2 \boldsymbol{\rho}_{\mathrm{c}}+\boldsymbol{\rho}_{\mathrm{w}}}-\left(\frac{\boldsymbol{\beta}_{\mathrm{c}}}{\boldsymbol{\beta}_{\mathrm{w}}}\right)\right]
$$

$\boldsymbol{\beta}_{\mathbf{c}}=$ molecular compressibility of those molecules present in that biological blood tissue complex.

$\boldsymbol{\rho}_{\mathbf{c}}=$ densities of molecules present in that biological blood tissue complex.

$\boldsymbol{\rho}_{\mathbf{w}}=$ densities of suspending medium present in the concerned biological blood tissue complex.

When light beam of Infrared LED were applied to detect the blood glucose based precise vibrational signatures for its respective concentration measurements, the famous Lambert-Beer law principles were considered to describe the phenomenon as follows [26,31-34]:

Where,

$$
\mathbf{A}(\mathbf{v})=-\log \mathbf{I}(\mathbf{v}) / \mathbf{I}_{0}(\mathbf{v})
$$

A= pattern of Absorption profile.

$\mathbf{v}=$ respective wave number.

$\mathbf{I}=$ light intensity of the adjacent medium.

$\mathbf{I}_{0}=$ light intensity after passing through the biological medium of blood tissue complex.

MUS-IR unit instrumental block diagram and its respective parts descriptions:

The comprehensive MUS-IR unit instrumental block diagrams for noninvasive blood glucose level predictions were depicted in Figure No.1. 
Five days daily sessions of noninvasive blood glucose level predictions based on amplitude modulated ultrasound and infrared technique over a healthy and diabetic subject

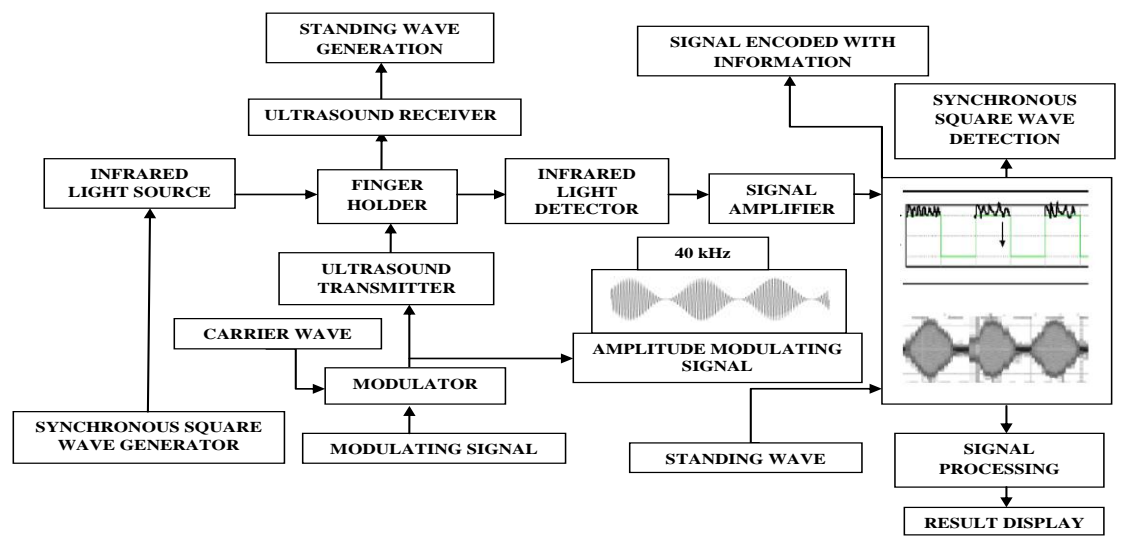

Figure No. 1: Illustrates schematic of the MUS-IR unit instrumental block diagram.

All the respective parts in that experimental setup were described below as follows:

Synchronous square wave generator: This part generates square wave pulses to the attached Infrared LED (Light Emitting Diode) light source.

Infrared light source: The operating wavelength of $940 \mathrm{~nm}$ had been utilized here for the experimental purposes. This unit connects to the finger holder unit.

The 940nm IR LED had been selected as it falls under the tissue optical window range from 700nm to $1100 \mathrm{~nm}$ [31-39]. Furthermore, the impact of interferences and hindrances from other optically similar sources like water, oxyhemoglobin and hemoglobin were sufficiently low in that particular wavelength zone often refereed as "tissue optical window" [35-39].

Modulating unit: This part had been attached with other two parts. First part belongs to carrier wave signal unit and the second one as modulating signal unit. The modulating unit performs the modulation functions to generate out amplitude modulated standing wave pulses in the direction of piezocrystal based ultrasonic transmitter unit.

Ultrasonic Transmitter (UST): A piezocrystal based ultrasound transmitter unit with $40 \mathrm{kHz}$ operating frequency had been supplied with amplitude modulated standing wave pulses to it. As seen in Figure No.2, in response to it, UST unit delivers the same amplitude modulated ultrasonic standing wave patterns towards finger holder unit.

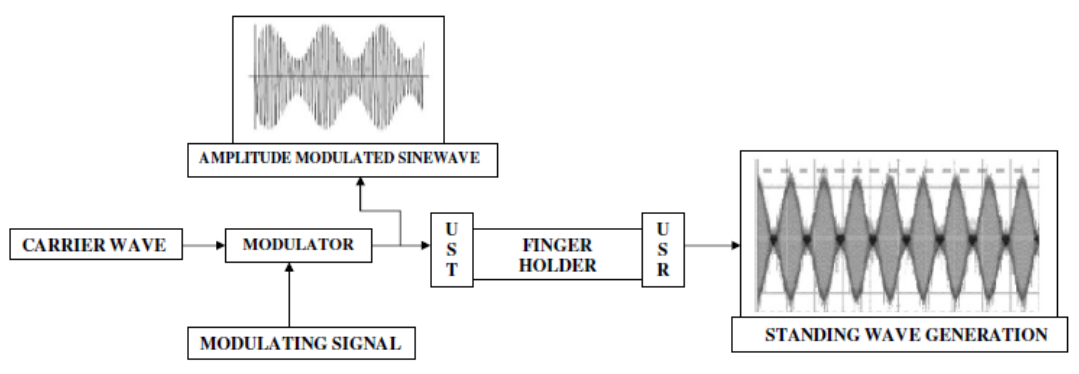

UST: ULTRASOUND TRANSMITTER

USR: ULTRASOUND RECEIVER

Figure No. 2: Represents the diagram of amplitude modulated ultrasonic unit of the MUS-IR setup.

Finger Holder Unit: The primary function of this unit as defined is to hold the finger in accurate geometrical position in order to minimize the various errors like motion artifacts, finger positioning based miscalculations, etc. The synchronous IR LED light source and the amplitude modulated Ultrasonic waves source were perpendicular to each other as compared to the finger positioning.

Ultrasonic Receiver (USR): Again, the piezocrystal based ultrasound receiver unit with $40 \mathrm{kHz}$ as working frequency had been utilized here. As seen from Figure No.2, the primary task of this unit is to check the pattern and orientations of the generated ultrasonic standing waves. Very important unit as the standing wave pattern based ultrasonic beam plays a significant role for noninvasive blood glucose level predictions. Both the UST and USR of $40 \mathrm{kHz}$ operating frequency were selected as it is safe and hazard free for its application over human beings [31-34].

Infrared (IR) detector: The blood glucose based precise vibrational signatures for its respective concentration measurements were picked up by this very sensitive IR detector unit 
Signal amplifier \& processing unit: The signal amplifications were performed in this unit after proper output signal acquisitions and noise filtrations. After that, the signals were processed suitably in the toolbox of MATLAB to extract the blood glucose level related information.

Result Display: The noninvasive blood glucose levels in $\mathrm{mg} / \mathrm{dl}$ were displayed through this unit.

Medical condition of the subjects:

A total of 02 subjects, from which one subject is normal healthy adults of age 28 years, height of $163 \mathrm{~cm}$ and weight around $72 \mathrm{~kg}$. The other diabetic subject is of age 35 years, height $165 \mathrm{~cm}$ and weight approximately 85 $\mathrm{kg}$. Both of them understood the experimental procedures. Written consents were obtained from them. Pilot study had been approved by the Institutional ethical Committee.

\section{Experimental procedures:}

The fasting, $2 \mathrm{hr}$ post prandial and random blood glucose levels of the normal and diabetic subjects were determined by the noninvasive blood glucose determination (MUS-IR) unit for five day daily sessions respectively. Similarly, for cross validation of the noninvasive blood glucose levels, Invasive glucometer (AccuChek Active from Roche Diagnostics) [40, 41] based readings were also obtained.

The noninvasive data, which had been obtained, were processed through the toolbox of MATLAB. In this method (modulated ultrasound and infrared techniques) the crest amplitude variation in FFT (Fast Fourier Transform) domain acts as a functional indicator for detecting blood glucose level changes during 5 days daily sessions from the normal and diabetic subjects.

\section{Results and Discussion}

The 5 days daily sessions of blood glucose detections in noninvasive manner had depicted us good potentiality in detecting blood glucose levels. The Table No. 1 and Graph No.1 reveals the data as obtained from the 5 days daily sessions of the normal subject. Similarly, the Table No.2 and Graph No.2 represent the 5 days daily sessions of the diabetic subject. Table No. 1,2 and Graph No. 1,2 represents the observational facts that the noninvasive blood glucose readings as obtained from the 5 days daily session of normal and diabetic subject were in good, acceptable correlation with the invasive blood glucose readings.

Clarke Error Grid analysis of 5 days daily sessions of the normal subject and diabetic subject were plotted in Graph No.3 and 4 respectively. All the data including normal and diabetic subject were again plotted (Clarke Error Grid analysis) in Graph No. 5 to produce the total evaluation of the noninvasive and invasive blood glucose level readings of 5 days daily sessions respectively. Usually, the Clarke Error Grid analytical system comprises of five zones such as Zone A, Zone B, Zone C, Zone D, Zone E respectively [42-46]. The data points in Zone A to B are medically acceptable and good correlating values in nature [42-46]. Moreover, the data points from Zone $\mathrm{C}$ to Zone $\mathrm{E}$ are medically irreverent as well as not acceptable [42-46]. For normal subject in Graph No.3, the Clarke Error Grid system represents the results for the data plots as follows: A zone $=86.6667 \%$, B zone $=13.3333 \%$, C zone $=00 \%$, D zone $=00 \%$, E zone $=00 \%$ respectively. Similarly, for diabetic subject in Graph No.4, the Clarke Error Grid system represents the results for the data plots as follows: A zone $=73.3333 \%$, B zone $=26.6667 \%, \mathrm{C}$ zone $=00 \%, \mathrm{D}$ zone $=00 \%, \mathrm{E}$ zone $=00 \%$ respectively. Again correspondingly, in Graph No.5, for both the normal and diabetic subjects, the Clarke Error Grid system represents the results for the data plots as follows: A zone $=80 \%$, B zone $=20 \%, \mathrm{C}$ zone $=00 \%$, D zone $=00 \%$, E zone $=00 \%$ respectively.

The output resultant values as obtained from these experiments show good resembles with our previous experimental results [31-34]. The important aspect for this technique comprises the significant utilization of ultrasonic waves which had been amplitude modulated in nature. Moreover, productive and standard acquisition of the propagated infra red light beam along with its critical signal processing procedures helps in its efficient detection for noninvasive blood glucose levels. The ultrasound and infrared technology combined together to yield high detecting sensitiveness along with accuracy and precisions.

Table No.1: Depicts the Blood Glucose Level $(\mathrm{mg} / \mathrm{dl})$ values as obtained by the noninvasive and invasive methods from the normal subject during 5 days daily sessions of fasting, post prandial, and random stages respectively

\begin{tabular}{|c|c|c|c|c|c|c|}
\hline \multirow{2}{*}{$\begin{array}{c}\text { 05 days } \\
\text { daily } \\
\text { sessions }\end{array}$} & \multicolumn{4}{|c|}{$\begin{array}{c}\text { Normal Subject Blood Glucose Level (BGL) } \\
\text { (mg/dl) }\end{array}$} \\
\cline { 2 - 7 } & $\begin{array}{c}\text { Fonsting Stage } \\
\text { method }\end{array}$ & $\begin{array}{c}\text { Invasive } \\
\text { method }\end{array}$ & $\begin{array}{c}\text { Noninvasive } \\
\text { method }\end{array}$ & $\begin{array}{c}\text { Invasive } \\
\text { method }\end{array}$ & $\begin{array}{c}\text { Noninvasive } \\
\text { method }\end{array}$ & $\begin{array}{c}\text { Invasive } \\
\text { method }\end{array}$ \\
\hline Day1 & 87 & 95 & 115 & 117 & 97 & 82 \\
\hline Day2 & 90 & 79 & 125 & 128 & 108 & 85 \\
\hline Day3 & 86 & 83 & 105 & 107 & 80 & 13 \\
\hline Day4 & 98 & 92 & 128 & 124 & 113 & 89 \\
\hline Day5 & 90 & 72 & 111 & 103 & 8 & 80 \\
\hline
\end{tabular}


Five days daily sessions of noninvasive blood glucose level predictions based on amplitude modulated ultrasound and infrared technique over a healthy and diabetic subject

Table No.2: Depicts the Blood Glucose Level (mg/dl) values as obtained by the noninvasive and invasive methods from the diabetic subject during 5 days daily sessions of fasting, post prandial, and random stages respectively

\begin{tabular}{|c|c|c|c|c|c|c|}
\hline \multirow{2}{*}{$\begin{array}{c}05 \text { days } \\
\text { daily } \\
\text { sessions }\end{array}$} & \multicolumn{4}{|c|}{$\begin{array}{c}\text { Diabetic Subject Blood Glucose Level (BGL) } \\
\text { (mg/dl) }\end{array}$} \\
\cline { 2 - 7 } & $\begin{array}{c}\text { Noninvasive } \\
\text { method }\end{array}$ & $\begin{array}{c}\text { Invasive } \\
\text { method }\end{array}$ & $\begin{array}{c}\text { Noninvasive } \\
\text { method }\end{array}$ & $\begin{array}{c}\text { Invasive } \\
\text { method }\end{array}$ & $\begin{array}{c}\text { Noninvasive } \\
\text { method }\end{array}$ & $\begin{array}{c}\text { Invasive } \\
\text { method }\end{array}$ \\
\hline Day1 & 128 & 143 & 207 & 261 & 149 & 137 \\
\hline Day2 & 138 & 119 & 220 & 195 & 156 & 116 \\
\hline Day3 & 130 & 119 & 212 & 177 & 153 & 169 \\
\hline Day4 & 137 & 101 & 223 & 193 & 169 & 179 \\
\hline Day5 & 139 & 123 & 214 & 197 & 128 \\
\hline
\end{tabular}

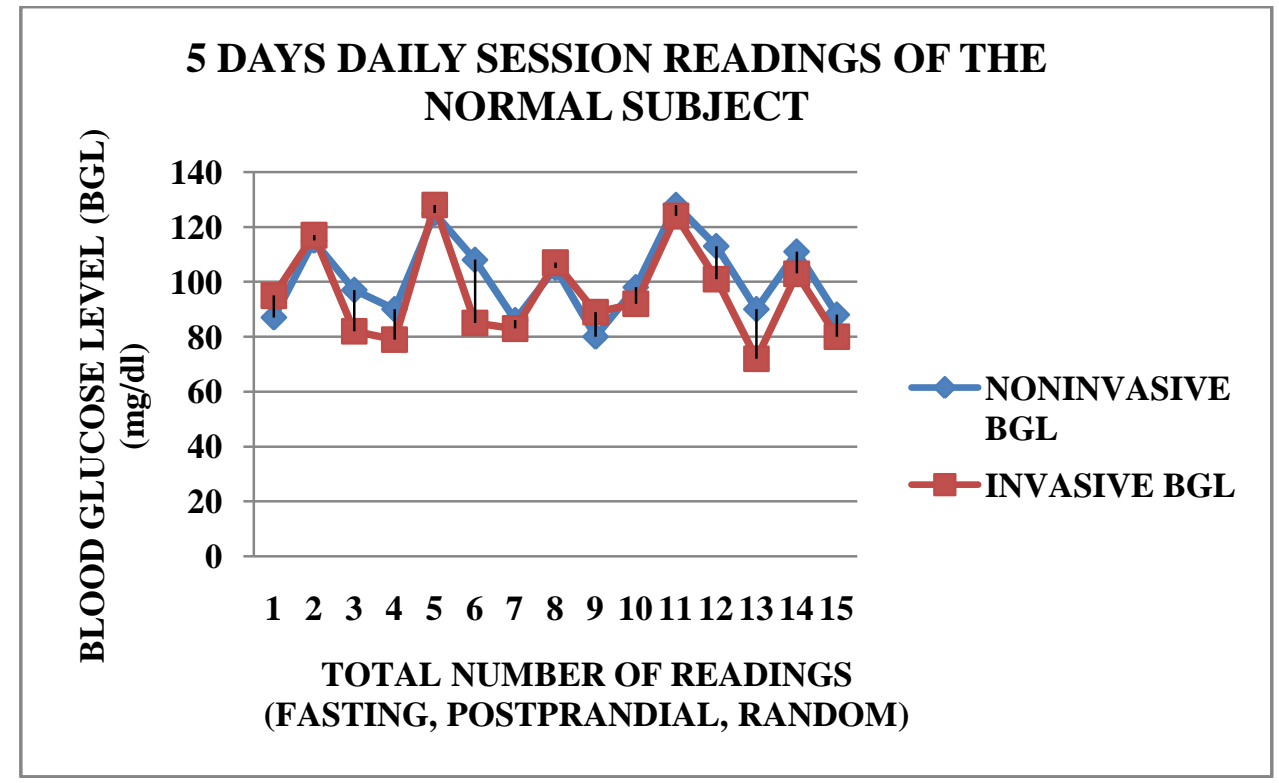

Graph No.1: Depicts the Blood Glucose Level values as obtained by the noninvasive and invasive methods from the normal subject during 5 days daily sessions of fasting, post prandial, and random stages respectively.

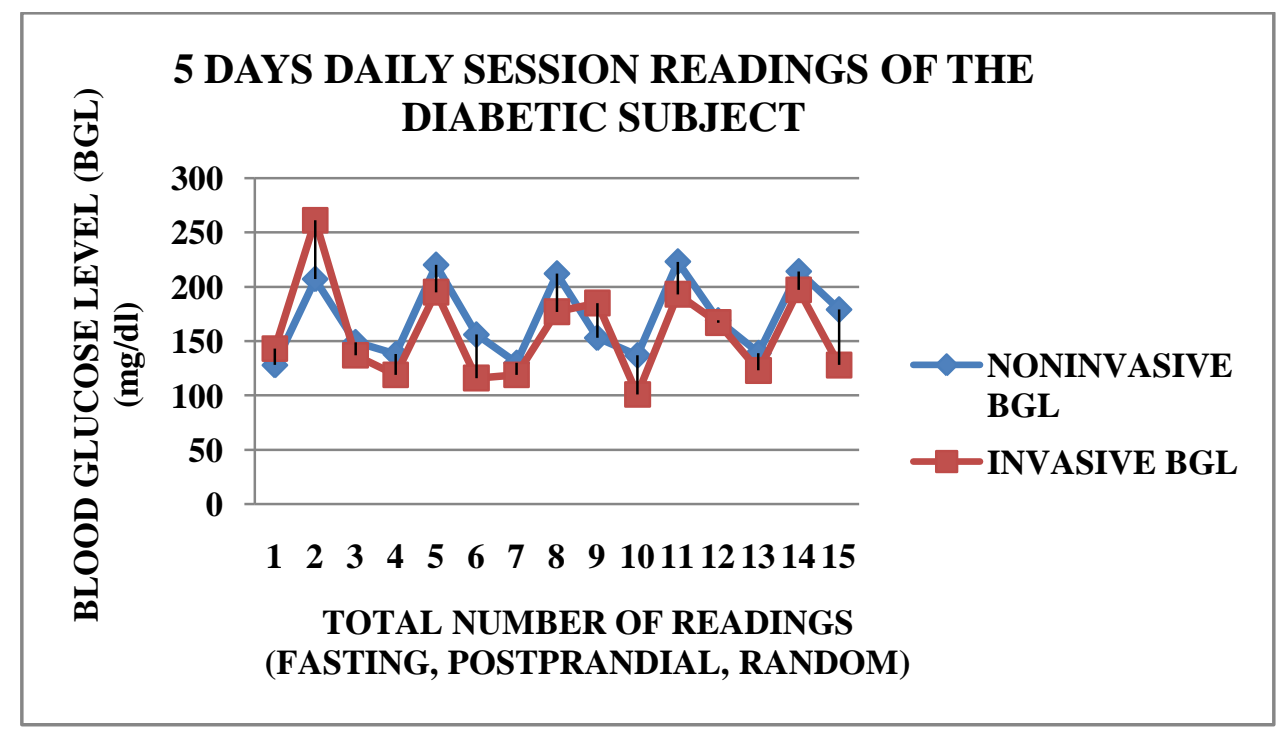

Graph No.2: Depicts the Blood Glucose Level values as obtained by the noninvasive and invasive methods from the diabetic subject during 5 days daily sessions of fasting, post prandial, and random stages respectively. 
Five days daily sessions of noninvasive blood glucose level predictions based on amplitude modulated ultrasound and infrared technique over a healthy and diabetic subject

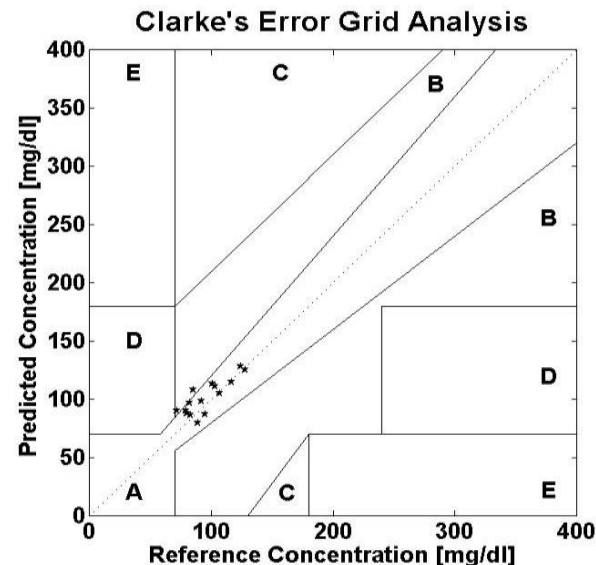

Graph No.3: Depicts the Clarke's Error Grid based Analysis of the Blood Glucose Level values as obtained by the noninvasive and invasive methods from the Normal subject during 5 days daily sessions of fasting, post prandial, and random stages respectively.

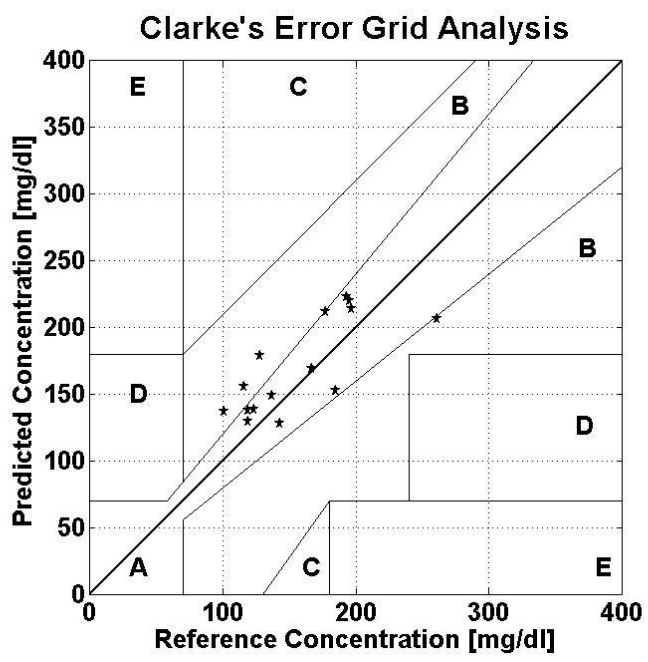

Graph No.4: Depicts the Clarke's Error Grid based Analysis of the Blood Glucose Level values as obtained by the noninvasive and invasive methods from the Diabetic subject during 5 days daily sessions of fasting, post prandial, and random stages respectively.

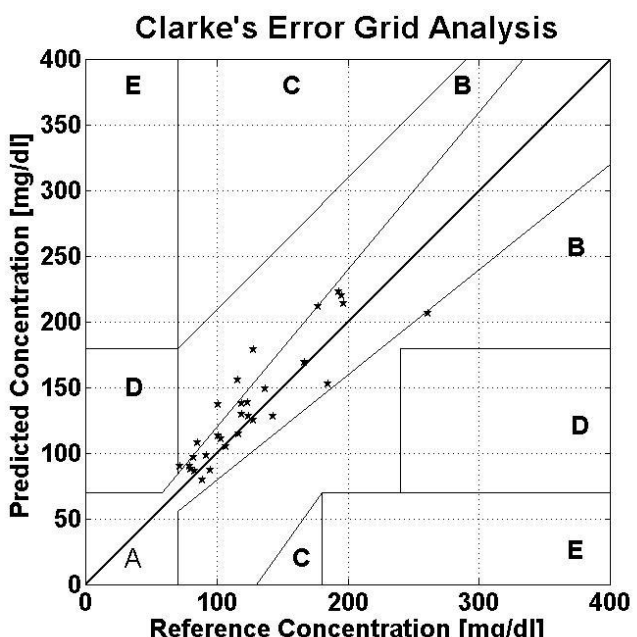

Graph No.5: Depicts the Clarke's Error Grid based Analysis of the Blood Glucose Level values as obtained by the noninvasive and invasive methods from both the subjects (Normal and Diabetic) during 5 days daily sessions of fasting, post prandial, and random stages respectively. 
The 5 days daily sessions based noninvasive method for blood glucose level predictions were obtained due to (a) the efficiency of the amplitude modulation based ultrasonic waves and infrared light technology to detect the glucose concentration irrespective of neighboring noises. (b) lower hindrances from the surrounding medium due to lower absorption profile of optically active oxyhaemoglobulin, haemoglobulin, water, etc molecules at the light spectral domain extending from 700nm to $1100 \mathrm{~nm}$ often referred as "tissue optical window" [35-38]. Our experimental work indicates towards the utilization of this new technique. It also emphasizes that the noninvasive technology based upon this technique (amplitude modulated ultrasound and infrared unit) could be developed to meet the demand for non invasive glucometer. Few erroneous signals were detected which may be due to human skin based pigmentations issues, variation in physiological phenomenon, change in blood flow patterns, motion related artifacts, surplus amount of light effect from the surroundings, etc.

\section{Conclusion}

We had shown the working efficiency of the MUS-IR unit for blood glucose level determinations in normal and diabetic subjects for five days in noninvasive style. A cross validation had been provided by comparing the values with invasive glucometer readings. A critical Clarke Error Grid based analysis for predicted (noninvasive) blood glucose concentration and reference (invasive) blood glucose concentration were performed and plotted here. All the data indicates towards the acceptable working efficiency and the potentiality of this new technique applied here.

\section{Acknowledgment}

The authors of the manuscript were grateful to the Coordinator, other faculty members, staffs of the School of Biomedical Engineering, IIT-(BHU), Varanasi for their valuable advice and timely assistance.

\section{References}

[1]. International Diabetes Federation, "IDF Diabetes Atlas," 6th edn. Brussels, Belgium: International diabetes Federation (2013). http://www.idf.org/diabetesatlas. (12/08/2014).

[2]. G. Danaei, M. M. Finucane, Y. Lu, G. M. Singh, M. J.Cowan, C. J. Paciorek, J. K. Lin, F. Farzadfar, Y. H.Khang, G. A. Stevens, M. Rao, M. K. Ali, L. M. Riley,C. A. Robinson, and M. Ezzati, 'National, regional, and global trends in fasting plasma glucose and diabetes prevalence since 1980: systematic analysis of health examination surveys and epidemiological studies with 370 countryyears and $2 \cdot 7$ million participants', Lancet 378, 31 (2011).

[3]. S. Wild, G. Roglic, A. Green, R. Sicree, and H. King, "Global Prevalence of Diabetes. Estimates for 2000 and projections for 2030." Diabetes Care, vol.27, 2003, pp 1047-1053

[4]. Peter J.Watkins, ABC of Diabetes, (Fifth Edition), London: BMJ Books, 2003.

[5]. http://diabetes.webmd.com/ (13/08/2014)

[6]. Tuchin V.V. (ed.) [Handbook of Optical Sensing of Glucose in Biological Fluids and Tissues]. CRC Press, Taylor \& Francis Group, London (2009).

[7]. A. Tura, A. Maran and G. Pacini, "Non-invasive glucose monitoring: Assessment of technologies and devices according to quantitative criteria", Diabetes Research and Clinical Practice, vol. 77, 2007, pp. 16-40

[8]. "Special issue on non-invasive glucose monitoring with optical technique", IEEE Leos Newsletter, April 1998.

[9]. J. Tenhunen, H. Kopola, and R. Myllyla, "Non-invasive glucose measurement based on selective near infrared absorption: requirements on instrumentation and special range," Measurement, vol.24, 1998, pp.173-177.

[10]. J.S. Maier, S.A. Walker, S. Fantini, M.A. Franceschini, and E. Gratton, "Possible correlation between blood glucose concentration and the reduced scattering coefficient of tissues in the near infrared," Opt. Lett., vol. 19, 1994, pp. $2062-2064$.

[11]. A.M.K. Enejder, T.G. Scecina, J. Oh, M. Hunter, W.-C. Shih, S. Sasic, G.L.Horowitz, and M. S. Feld, "Raman spectroscopy for noninvasive glucose measurements,"J.Biomed. Opt., vol. 10, 2005, 031114.

[12]. J. Lakowicz and B. Maliwal, "Optical sensing of glucose using phase modulation fluorimetry", Anal.Chim.Acta, vol.271, 1993, pp.155-164.

[13]. S-j Yeh, C. F. Hanna, S. Kantor, et al., "Differences in thermal optical response between intact diabetic and nondiabetic human skin," Proc. SPIE, vol.4958, 2003, pp. 213-224.

[14]. S. Bockle, L. Rovati, and R.R. Ansari, "Polarimetric glucose sensing using the Brewster-reflection off-eye lens: theoretical analysis," Proc. SPIE, vol. 4624, 2002, pp. 160-164.

[15]. C. Chou, C.Y. Han, W.C. Kuo, Y.C. Huang, C.M. Feng, and J.C. Shyu, "Noninvasive glucose monitoring in vivo with an optical heterodyne polarimeter," Appl. Opt., vol. 37, 1998, pp. 3553-3557.

[16]. V.L. Alexeev, A.C. Sharma, A.V. Goponenko, S. Das, I.K. Lednev, C.S. Wilcox, D.N. Finegold, and S.A. Asher, "High ionic strength glucose sensing photonic crystal," Anal. Chem., vol. 75, 2003, pp. 2316-2323.

[17]. Z. Zhao, Pulsed Photoacoustic Techniques and Glucose Determination in Human Blood and Tissue, doctoral thesis, University of Oulu, Finland, 2002.

[18]. H.S. Ashton, H.A.MacKenzie, P. Rae, Y.C. Shen, S. Spiers, et al., "Blood glucose measurements by photoacoustics," CP463 Photoacoustic and Photothermal Phenomena: 10th International Conference, 1999, pp. 570-572.

[19]. K.V. Larin, M.S. Eledrisi, M. Motamedi, R.O. Esenaliev, "Noninvasive blood glucose monitoring with optical coherence tomography: a pilot study in human subjects," Diabetes Care, vol. 25, no. 12, 2002, pp. 2263-2267.

[20]. L.Zhu, J.Lin, B.Lin, H.Li., "Noninvasive blood glucose measurement by ultrasound-modulated optical technique",Chinese Optical Letters, 11(2),2013,pp.021701-1 to 021701-5.

[21]. Orna Amir, Daphna Weinstein, Silviu Zilberman, Malka Less, Daniele Perl-Treves,Harel Primack, Aharon Weinstein, Efi Gabis,Boris Fikhte,Avraham Karasik, "Continuous Noninvasive Glucose Monitoring Technology Based on Occlusion Spectroscopy", J Diabetes Sci Technol Vol 1, Issue 4, July 2007,pp.463-469. 
[22]. O. Khalil, "Noninvasive glucose measurement technologies: an update from 1999 to the dawn of the new Millennium," Diabetes Technol. Ther., vol. 6, no. 5, 2004, pp. 660-697.

[23]. Ter Haar, G. and S.J. Wyard, Blood cell banding in ultrasonic standing wave fields: A physical analysis. Ultrasound in Medicine and Biology, 1978. 4(2): p. 111-123.

[24]. Md.K.Chowdhury, A.Srivastava, N.Sharma, S.Sharma, "Challenges \& Countermeasures in Optical Noninvasive Blood Glucose Detection”, International Journal of Innovative Research in Science, Engineering and Technology (IJIRSET), Vol.2, issue 1,Jan 2013,pp.324-329.

[25]. A.Srivastava, Md.K.Chowdhury, S.Sharma, N.Sharma, "Blood Glucose Monitoring Using Non Invasive Optical Method: Design Limitations and Challenges", International Journal of Advanced Research in Electrical, Electronics and Instrumentation Engineering (IJAREEIE), Vol. 2, issue 1, Jan 2013, pp.615-620.

[26]. S. Radel , M. Brandstetter, B.Lendl, 'Observation of particles manipulated by ultrasound in close proximity to a cone-shaped infrared spectroscopy probe', Ultrasonics 50 (2010), pp.240-246.

[27]. W. Terence Coakley, 'Ultrasonic separations in analytical biotechnology', Trends in Biotechnology (1997), pp. 506-511.

[28]. L.V. King, 'On the acoustic radiation pressure on spheres', Proceedings of the Royal Society of London (1934), pp.212-240. A147.

[29]. K. Yosioka, Y. Kawasima, 'Acoustic radiation pressure on a compressible sphere', Acustica 5 (1955), pp.167-173.

[30]. F. Petersson, A. Nilsson, C. Holm, H. Jonsson and T. Laurella, 'Separation of lipids from blood utilizing ultrasonic standing waves in microfluidic channels', The Analyst, The Royal Society of Chemistry,(2004),129,pp.938-943. DOI: 10.1039/b409139f.

[31]. Md. K. Chowdhury, A. Srivastava, N. Sharma, \& S.Sharma, 'The influence of blood glucose level upon the transport of light in diabetic and non-diabetic subjects'. International Journal of Biomedical and Advance Research, 4(5) (2013), pp.306-316. doi:10.7439/ijbar.v4i5.357.

[32]. A. Srivastava, Md. K. Chowdhury, S. Sharma, N. Sharma, 'Optical Clearance Effect Determination of Glucose by near Infrared Technique: An Experimental Study using An Intralipid Based Tissue Phantom', International Journal of Advances in Engineering \& Technology (IJAET), Volume 6 Issue 3, pp. 1097-1108, July 2013.

[33]. Md. Koushik Chowdhury, Anuj Srivastava, Shiru Sharma, Neeraj Sharma, "The potential application of amplitude modulated ultrasound with Infrared Technique for blood glucose level determination in non invasive manner". Biomedical and Pharmacology Journal, Vol.7 (1), pp.195-206 (2014).

[34]. A.Srivastava, Md.K.Chowdhury, S.Sharma, N.Sharma, "Measurement of Glucose Concentration using Amplitude Modulated Ultrasound with Infrared Technique in Intralipid Phantoms and Human Whole Blood mixed intralipid phantom of Healthy and Diabetic Subjects", Biosciences Biotechnology Research Asia, Vol.11, No.2, pp.593-602 (2014).

[35]. K. Konig, "Multiphoton microscopy in life sciences", Journal of Microscopy, vol. 200-2, 2000, pp. 83-104.

[36]. J. Tenhunen, H. Kopola, and R. Myllyla, "Non-invasive glucose measurement based on selective near infrared absorption: requirements on instrumentation and special range," Measurement, vol. 24, 1998, pp. 173-177.

[37]. O.W. Assendelft, Spectrophotometry of Hemoglobin Derivates, Royal Vangorcum Ltd., Assen, 1970.

[38]. Y.Mendelson, "Pulse oximetry: theory and applications for noninvasive monitoring," Clin. Chem., vol. 38, 1992 , pp.1601-1607.

[39]. D. J. Cox, W. L. Clarke, L. Gonder-Frederick, S. Pohl, C. Hoover, A. Snyder, L. Zimbelman, W. R. Carter, S. Bobbitt, and J. Pennebaker, "Accuracy of perceiving blood glucose in IDDM," Diabetes Care, vol. 8, no.6, pp. 529-536, 1985.

[40]. Brian Hill, "Accu-Chek Advantage: Electrochemistry for Diabetes Management", Current Separations, pp.45-48. http://www.currentseparations.com. (12/08/2014)

[41]. https://www.accu-chek.com/index.html. (12/08/2014)

[42]. W. L. Clarke, L. A. Gonder-Frederick, W. Carter, and S. L. Pohl, "Evaluating clinical accuracy of systems for self-monitoring of blood glucose, "Diabetes Care, vol. 10, no. 5, pp. 622-628, 1987.

[43]. A. Maran et al. "Continuous Subcutaneous Glucose Monitoring in Diabetic Patients" Diabetes Care, Volume 25, Number 2, February 2002

[44]. B.P. Kovatchev et al. "Evaluating the Accuracy of Continuous Glucose Monitoring Sensors" Diabetes Care, Volume 27, Number 8, August 2004.

[45]. E. Guevara and F. J. Gonzalez, Prediction of Glucose Concentration by Impedance Phase Measurements, in MEDICAL PHYSICS: Tenth Mexican Symposium on Medical Physics, Mexico City (Mexico), 2008, vol. 1032, pp.259-261.

[46]. E. Guevara and F. J. Gonzalez, Joint optical-electrical technique for noninvasive glucose monitoring, REVISTA MEXICANA DE FISICA, vol. 56, no. 5, pp. 430-434, Sep. 2010. 